\title{
Untethering sparteine: 1,3-diamine ligand for asymmetric synthesis in water
}

\author{
Fanyun Zeng ${ }^{1} \dagger$, Chen Chen ${ }^{1} \uparrow$, Liu Liu ${ }^{1} \dagger$, Yifan $\operatorname{Li}^{1} \uparrow, \mathrm{Bo} \mathrm{Li}^{2}$, Guotao $\operatorname{Lin}^{1}$, Shihao \\ Huang ${ }^{1}$, Mingyu Zhang ${ }^{1}$, Yuting Yan ${ }^{1}$, Yuan Zhang ${ }^{1}$, Yanfeng Dang ${ }^{2 *}$, Jianhui Huang ${ }^{1 *}$
}

\section{Affiliations:}

${ }^{1}$ Tianjin Key Laboratory for Modern Drug Delivery \& High-Efficiency; School of Pharmaceutical Science and Technology, Tianjin University; Tianjin, 300072, China.

${ }^{2}$ Tianjin Key Laboratory of Molecular Optoelectronic Science, Department of Chemistry, School of Science, Tianjin University; Tianjin, 300072, China.

*Corresponding author. Email: yanfengdang@tju.edu.cn; jhuang@tju.edu.cn.

$\dagger$ These authors contributed equally to this work.

\$n memory of Professors Jonathan Williams, Peter Beak and Nathaniel Finney.

\begin{abstract}
Chiral ligands are the toolbox for asymmetric synthesis to access 3D molecular world. Enabling efficient asymmetric reaction in water is a big challenge. As moisture/air stable and strong binding moieties, amines, compared to imine and phosphine ligands, are ideal candidates to accommodate asymmetric transformations in water. Known amine ligands like Proline analogues and Cinchona alkaloids showed excellent asymmetric induction. Sparteine, an alkaloid studied originated in 1968, had never been considered as a privileged catalyst due to its structure defection which led to poor reaction compatibility and unsatisfactory stereoselectivity. Here, we report the design of a chiral diamine catalyst untethering one of the sparteine rings. The diamine catalyst was easily accessed in two steps on 100 gram-scale. This chiral ligand was proved to be efficient for addition reactions in water providing products with excellent yields and enantiomeric ratios. This pluripotent catalyst has also shown good reactivity/enantioselectivity under organocatalysis, $\mathrm{Cu}$ and $\mathrm{Pd}$-catalysed conditions. We anticipate that the ligand would allow further development of other catalysts for important yet challenging green stereoselective transformations.
\end{abstract}

From feedstock to commodity chemicals and further to biological and pharmaceutical active ingredients, asymmetric synthesis has paved the way to access molecules covering spacious chemical space and structure diversity/complexity ${ }^{1}$. The green synthesis of chiral molecules is important especially on industrial scale production. In asymmetric synthesis, chiral catalysts have broad applications in various reaction pathways. Different from bio-catalysts ${ }^{2}$, commonly recognized privileged chiral catalysts $^{3,4}$ as summarized by Jacobsen and Zhou shown in Fig. 1A, the chiral ligands were listed in chronological order. From 1960s, natural products were firstly studied. Imine and phosphine ligands bloomed at the end of $20^{\text {th }}$ century, hundreds of analogues had been developed. There has been no new ligand class recruited in recent 20 years. Future ligand design meeting the need of green chemistry is the ultimate desire ${ }^{5}$. 
Comparing BINAP, Pyrox, Proline analogue Barbas catalyst and sparteine, as listed in Fig. 1B, four key factors of ligand design were demonstrated. BINAP, due to its sensitivity to air and moisture, Pyrox, weak binding, was not suitable for asymmetric reactions in water. Amine-containing ligands having strong binding and stability are potential candidates for reactions in water. Natural occurring products, Proline and Cinchona alkaloids are widely used in asymmetric synthesis and recognized as privileged catalysts. Sparteine, with both enantiomers, $(+)$-sparteine and $(-)$-sparteine commercially available, was used in asymmetric synthesis since 1970 s by Noyori ${ }^{6}$ and Trost ${ }^{7}$. It has also been used in $\mathrm{Li}^{8-14}, \mathrm{Mg}^{15}, \mathrm{Ni}^{16}, \mathrm{Cu}^{17,18}, \mathrm{Zn}^{19}$ and $\mathrm{Pd}^{20-23}$ mediated reactions. However, the reaction conversion and enantioselectivity was poor and required high catalyst loading. Sparteine had never been recognized as a privileged catalyst. From structure perspective, as shown in Fig. 1B, sparteine compared with the other three catalysts, the skeleton is over-rigid and both rings attached on bispidine was sterically less favored for catalytic reactions. To avoid the steric congestion, O'Brien diamine (Fig. 2E, L2) was prepared and was only used in carbanion chemistry. Barbas catalyst, the sterically least hindered $N-\mathrm{H}$ diamine, was considered as a model in our design.

A

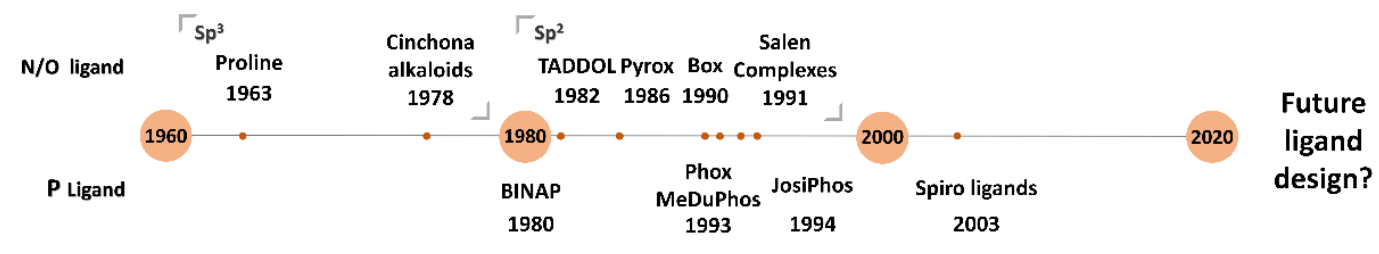

B

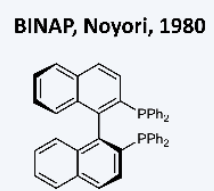

Pyrox, Brunner, 1986

Proline analogue Barbas' catalyst, 2004

Sparteine, Noyori, 1968

(2)

Balance of rigidity and flexibility

Binding affinity

Air/moisture sensitivity

Analogue accessibility

$\frac{\square}{\square}$
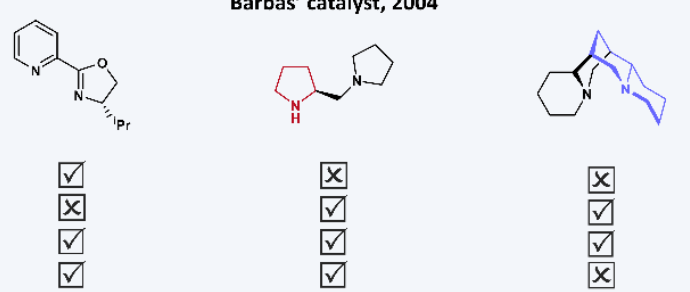

$\frac{\square}{\square}$
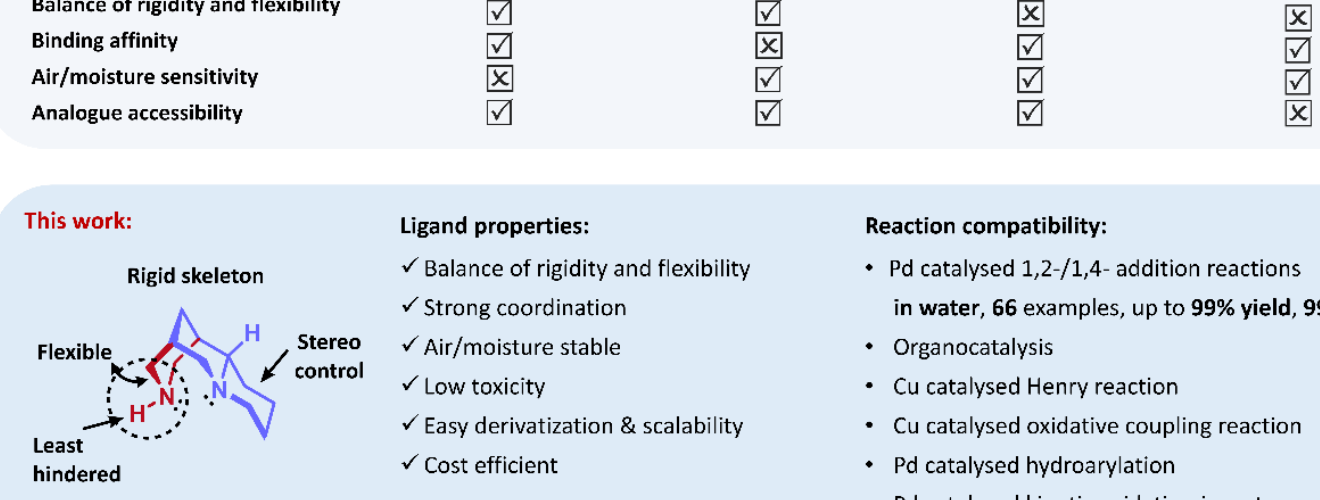

Reaction compatibility:

- Pd catalysed 1,2-/1,4- addition reactions

in water, 66 examples, up to $99 \%$ yield, 99:1 er.

- Organocatalysis

- Cu catalysed Henry reaction

- Cu catalysed oxidative coupling reaction

- Pd catalysed hydroarylation

- Pd catalysed kinetic oxidation in water

Fig. 1 History of well-known chiral ligands. (A) Privileged chiral ligands (B) Rational ligand design and asymmetric syntheses using chiral $\mathrm{N}-\mathrm{H}$ diamine ligand.

Here, we report a computer-aided ligand design combining the features of flexible Barbas catalyst and over-rigid sparteine to access a low toxic $\mathrm{N}-\mathrm{H}$ diamine for enantioselective catalysis in water. The selected asymmetric reactions have shown that the chiral ligand has high reactivity and stereoselectivity with broad applications on a number of reactions in green and organic solvents. The allowance of a family of 
ligands with functionality at various positions was also demonstrated on asymmetric addition reaction with toxicity evaluations.

Quantum mechanical computations were applied to engineer the sparteine scaffold to achieve better performance. Intending the scaffold optimization to be more general, we sought to tailor the ligand environment for reaction events in the first coordination sphere, which we recognize as a major challenge, by focusing on a representative inner-sphere process by Pyrox-Pd catalysis ${ }^{24}$, i.e. the asymmetric addition of $N$-Ts imine with $\mathrm{PhB}(\mathrm{OH})_{2}$ (Fig. 2A). Computational studies led us to establish a plausible pathway consisting of transmetalation (TS1), migratory insertion (TS2), and protonation $(\mathbf{T S 3})^{25}$. The resulting free energy profile drew our attention to the ratelimiting and enantio-controlling migratory insertion step. Two clues for ligand design were implicated by an analysis of TS2. First, this sterically congested transition state, displaying multiple ligand-substrate repulsions (Fig. 2B), could be stabilized by decreasing peripheral hindrances (e.g. rings 1 and 2), providing a means of accelerating the catalytic process. Second, the computed stereoisomeric transition states suggest that the barrier difference of TS2a vs TS2b is significantly larger than TS2a' vs TS2b' (Fig. 2C). We reasoned that an arrangement where the more anisotropic $\mathrm{N}-\mathrm{Ts}$ imine is placed on the sterically biased right-hand side of Sparteine (i.e. as in TS2a/TS2b; see Fig. 2D for a steric contour) would be desirable for maximizing stereodifferentiation.

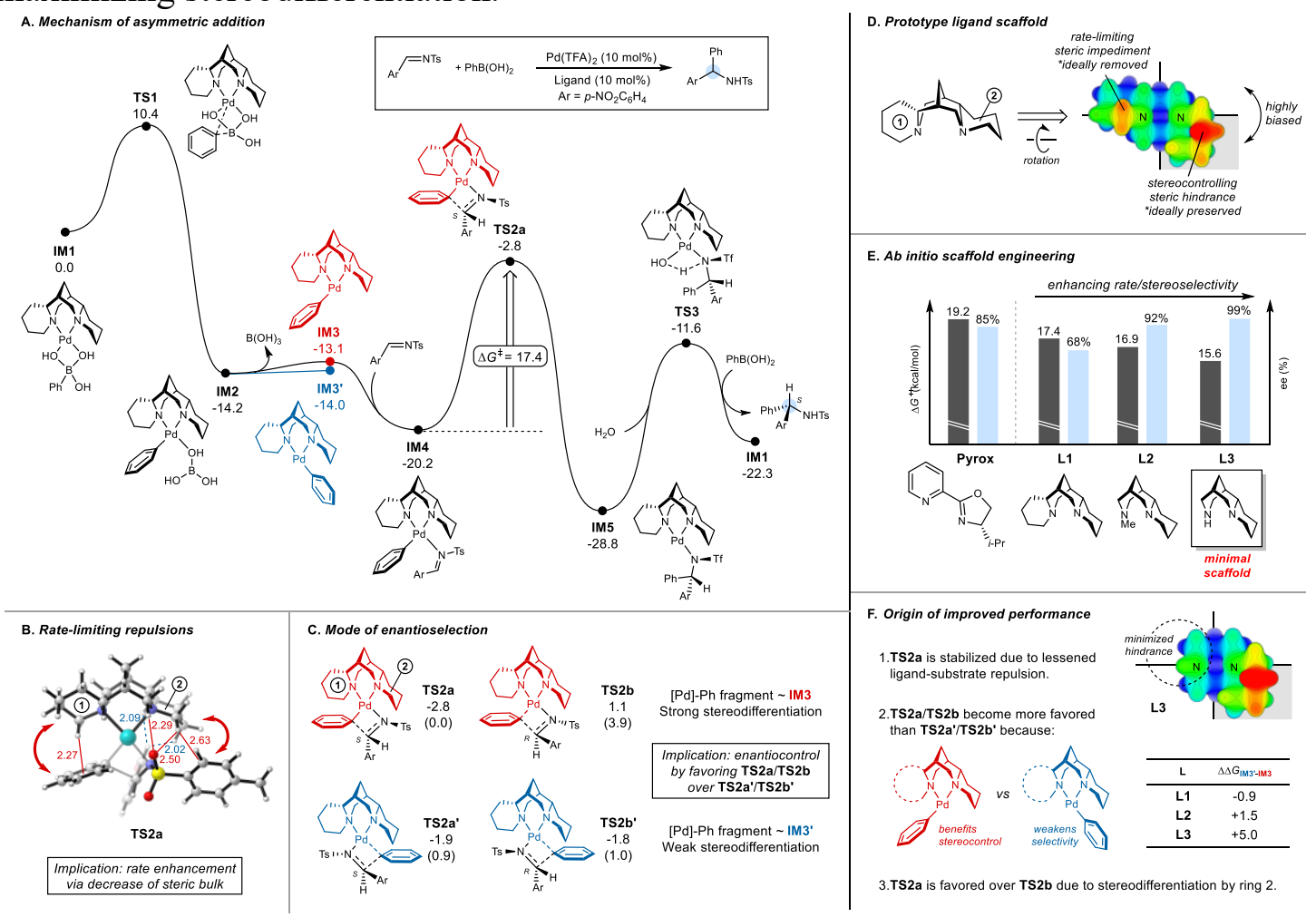

Fig. 2. Computational ligand design. (A) Mechanism of $\mathrm{Pd} /$ sparteine-catalysed asymmetric addition. (B) Rate-limiting steric repulsions. (C) Mode of enantioselection. (D) Prototype ligand scaffold. (E) Ab initio scaffold engineering. (F) Origin of improved performance. Free energies are shown in $\mathrm{kcal} / \mathrm{mol}$, relative values in parentheses. More computational details are provided in the Supporting Information.

We tested our hypotheses by computationally assessing the influence of removing ring 1 from sparteine (Fig.s 2D and 2E). To our delight, it was demonstrated by L1-L3 that a significant improvement can be realized by our backbone engineering. The 
computationally derived ligand $\mathbf{L 3}$, which preserves the rigid bispidine core but can better accommodate and exert stereocontrol over the reactive fragments (Fig. 2F), gives a low barrier of $15.6 \mathrm{kcal} / \mathrm{mol}$ and an excellent ee of $99 \%$, both superior to the prototype $\mathbf{L} 1$ as well as the Pyrox privileged ligand. The effectiveness of $\mathbf{L} 3$ implies a strong stereodiscrimination over inner-sphere transformations from a minimal chiral scaffold, illustrating the promising potential of sparteine-family ligands for a broader scope of asymmetric catalysis.

Based on the computational studies above as well as rationale from Barbas ${ }^{26}$, Kozlowski and Gaunt on $N-\mathrm{H}$ containing amine ligands ${ }^{27,28}$, O'Brien diamine $\mathbf{L 2}^{29,30}$ and $\mathrm{N}-\mathrm{H}$ diamine $\mathbf{L} 3$ have been prepared with modified procedures based on the literature precedence ${ }^{31,32}$. (see SI, Scheme S1) Starting from commercially available alkaloid (-)-cytisine ${ }^{33}$, both diamines were successfully prepared in 100 gram-scale and were ready for the further assessments on asymmetric reaction under sustainable conditions. The structure of bench-stable $\mathbf{~} \mathbf{3} \cdot \mathbf{H C l}$ salt was unambiguously determined by X-ray single crystal diffraction (CCDC No.2116108). Reactions of cyclic and acyclic imines with 4-methoxylphenyl boronic acid in trifluoroethanol have been studied first. Even though in the literature, these diamine ligands were not considered as good ligands for reactions out of organolithium field ${ }^{34,35}$. Both of diamines $\mathbf{L} 2$ and L3 have provided excellent reactivity and enantioselectivity for all of the four reactions comparing to the moderate enantiomeric ratios generated from $(+)$-sparteine $\mathbf{L 1}$ as shown in Fig. $3 \mathrm{~A}^{36}$. Interestingly, for the addition to sterically hindered $\mathrm{N}$ sulfonyl ketimines, ligand $\mathbf{L 3}$ provided the corresponding sulfonamide $\mathbf{2}$ in $90 \%$ yield with 96:4 er while $N-$ Me diamine ligand $\mathbf{L} 2$ provided the product in $67 \%$ yield with reduced er (69:31), while the use of $(+)$-sparteine $\mathbf{L} \mathbf{1}$ gave diminished enantioselectivity (50:50 er).

To test our hypothesis, in water, the same reaction using known privileged catalysts such as BINAP L4, Phox $\mathbf{L 5}^{37-39}$, Pyrox $\mathbf{L 6}{ }^{40}$ as well as Proline $\mathbf{L} 7^{41}$ and quinine $\mathbf{L 8}$, together with O'Brien diamine $\mathbf{L} 2, N-\mathrm{H} \mathbf{L} 3$ were carried out. The desired product 1 was obtained with yields and er.s shown in Fig. 3B. BINAP L4 provided product 1 with good stereoselectivity of 95:5 er but moderate yield of $40 \%$ due to the oxidation of phosphine during the reaction; Phox $\mathbf{L 5}$ gave the corresponding product 1 with good yield and er; Pyrox L6, Proline $\mathbf{L} 7$ and quinine $\mathbf{L 8}$ were not efficient chiral catalysts for this reaction in water even with moderate to good reaction yields. Whereas diamine $\mathbf{L} 2$ provided the sulfonamide 1 with good yield and good er, $N-\mathrm{H}$ diamine $\mathbf{L 3}$ gave the product with nearly quantitative yield with excellent er. (Fig. 3B)

With the conditions in hands, we have examined the reactions employing diamine $\mathbf{L} 2$ or $\mathrm{N}-\mathrm{H}$ diamine L3. Reactions of arylboronic acids with 5-membered cyclic sulfonyl imides were also very successful in water. A group of representative sulfonamides 513 have been prepared with both $N-\mathrm{H}$ and $N-$ Me ligands under similar conditions. Very encouragingly, among all the examples demonstrated, $N-\mathrm{H}$ diamine $\mathbf{L 3}$ and O'Brien diamine $\mathbf{L} 2$ have shown excellent reactivity and stereoselectivity, and all of the desired products were obtained in excellent yields and enantiomeric ratios. (Fig. $3 \mathrm{C})$ 


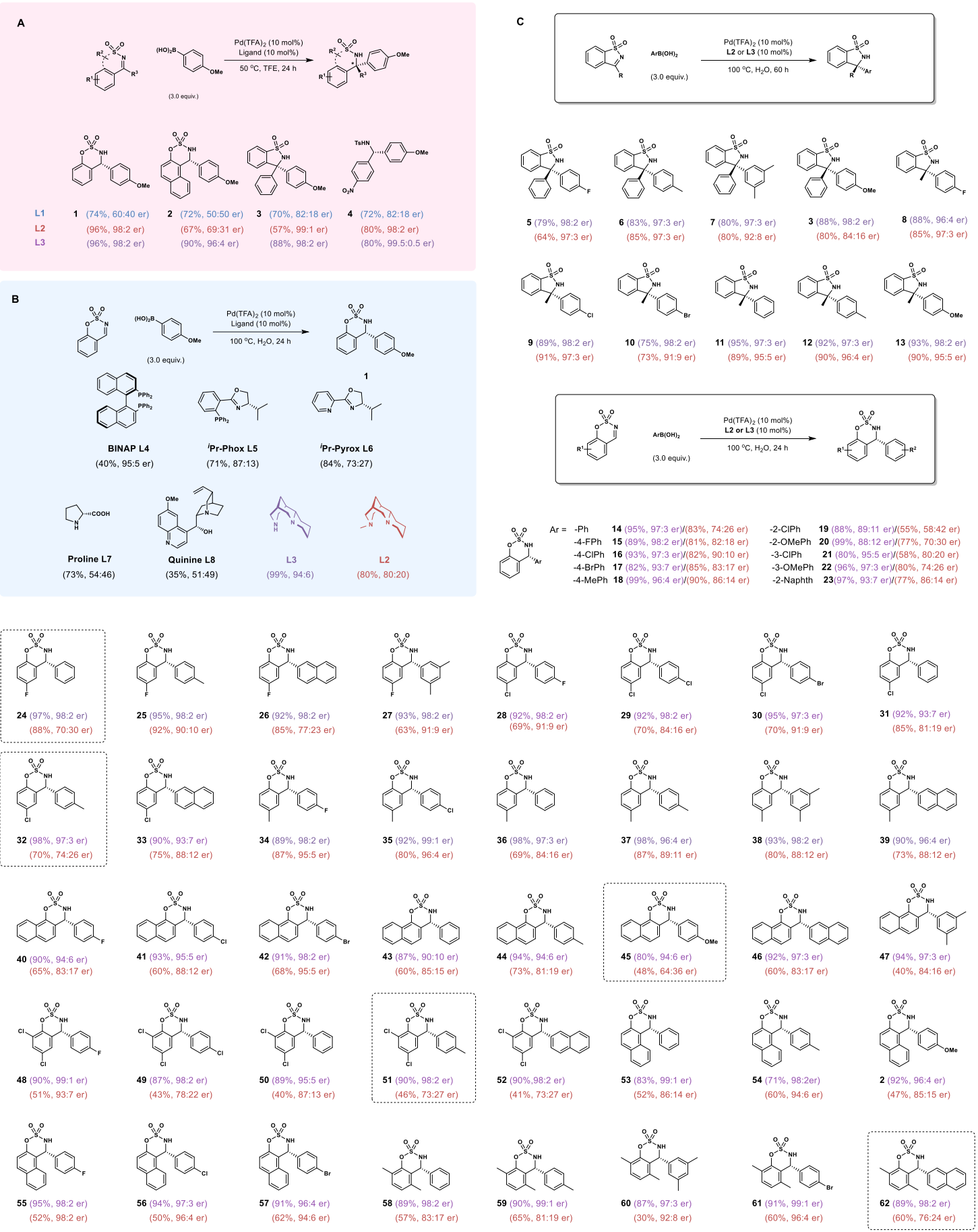

Fig. 3. Green synthesis of chiral sulfonamides. (A) Ligand screening in organic solvent. (B) Ligand screening in water. (C) Reaction scope with chiral diamine ligands $\mathbf{L} 2$ or $\mathbf{L} 3$ in water.

Reactions of arylboronic acids with 6-membered cyclic $N$-sulfonyl ketimines were also fruitful. A large number of corresponding sulfonamides have been prepared with either diamines $\mathbf{L 2}$ or L3. (Fig. 3C) We were pleased to find in all the cases, $N-\mathrm{H}$ diamine $\mathbf{L} 3$ gave higher yields with higher enantiomeric ratios comparing to $N-\mathrm{Me}$ diamine L2. Particularly for reactions with sterically hindered nucleophiles such as naphthyl boronic acid, the er values for $N$-Me were generally much lower than the other reactions. (see selected examples highlighted in boxes) The formation of sulfonamide 19 was very positive using $N-\mathrm{H}$ diamine instead of $N-$ Me ligand while 
the er values of reactions with two ligands were 11:89 and 42:58 respectively. A large number of representative examples have been demonstrated and the corresponding chiral sulfonamides were obtained in good yields and enantioselectivities.

Not only the 1,2-addition reaction, the reactions of arylboronic acid under Pd catalysed conditions were also very successful for the Michael addition on enones (Fig. 4A). The corresponding products were also obtained in good yields and ers. Comparing to the known ligand ${ }^{i}$ Pr-Pyrox (70:30 er) reported by Stoltz ${ }^{25}$, our ligand provided the product with higher er even with a simple skeleton (13 atoms) before the introduction of sterically enhancing group.

To evaluate the ligand further, a number of representative reactions including organocatalysis, $\mathrm{Cu}$-catalysed Henry reaction and oxidative coupling reaction; Pdcatalysed hydroarylation and kinetic oxidation reaction were examined. Under unoptimized conditions, the reaction provided the corresponding products with good yields and good enantiomeric ratios. As shown in Fig. 4B, organocatalysis under neat conditions, the desired chiral alcohol 71 was obtained in $70 \%$ yield with $83: 17 \mathrm{dr}$ and 99:1 $\mathrm{er}^{42}$. Binol derivative $\mathbf{7 3}$ was also successfully prepared in 71\% yield and 96:4 $\mathrm{er}^{18}$. More interestingly, reaction of iodobenzene with norbornene, in the presence of Pd catalyst and diamine ligand $\mathbf{L 3}$, the desired phenyl substituted norbornane $\mathbf{7 7}$ was prepared in 95:5 er whereas in the literature, the best enantioselectivity was achieved under Zhou's conditions using Quinox ligand at 87:13 $\mathrm{er}^{43}$. (Fig. 4B, eq. 4) A standard Henry rection using aldehyde 69 with nitromethane provided the corresponding chiral alcohol 74 in 77\% yield with 90:10 er in ethanol ${ }^{44}$. In water, under conditions similar to Sigman and Stoltz reported in organic solvent 20 years $\mathrm{ago}^{20,21}$, the kinetic oxidation reaction was also successful and alcohol 78-R was obtained in 43\% yield with 76:24 er.
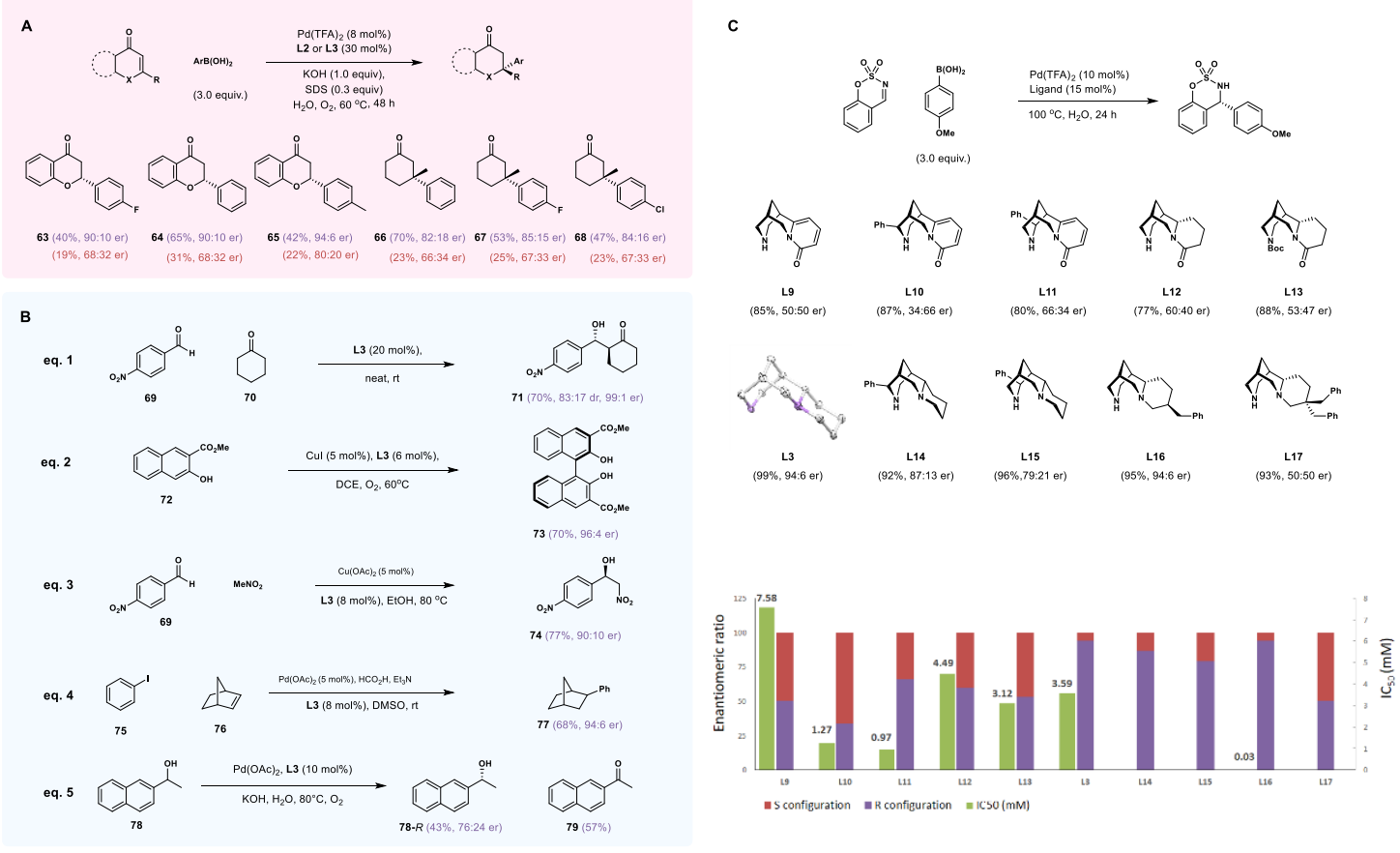

Fig. 4. Ligand evaluation on various mechanistically irrelevant reactions. (A) Asymmetric Michael addition in water. (B) Miscellaneous reactions using diamine ligand L3. (C) Diamine ligand modifications, evaluations and toxicity studies against HEK293T cells. 
Similar chiral diamine ligands within the family have been prepared and evaluated against a model reaction in water. Under standard conditions, ligands L3 and L15 have shown similarly high asymmetric induction, while the introduction of groups on (-)cytisine derivatives provided sulfonamide 1 with good yields but low er.s. Ligands such as L8, L10 and L12 have shown reduced enantioselectivities; L9 and L10 have shown opposite asymmetric induction due to the substituent on the other site of (-)cytisine.

With regard to green catalysts, the toxicity studies were also carried out for selected chiral $\mathrm{N}-\mathrm{H}$ diamines in order to reveal the green profiles for the future ligand development. Using HEK293T cell, these ligands have been evaluated. Diamine L8, as a smoking cessation agent, showed the lowest toxicity among these diamines ${ }^{45}$ (Fig 4C, green bar). Ligands L3, L11, L12 showed similarly low toxicity to (-)-cytisine even though $\mathbf{L 1 1}$ and $\mathbf{L 1 2}$ provided the reactions with low er.s. Unfortunately, ligand L15 with good reactivity and enantioselectivity has shown over 200 times higher toxicity comparing to (-)-cytisine (see SI table S1). It is delightful that our pluripotent chiral diamine ligand $\mathbf{L 3}$ showed low cytotoxicity to become a possible ligand in green chemistry.

We have developed a new class of chiral $\mathrm{N}-\mathrm{H}$ diamine ligands for catalysis. The results highlight the substantial scope of the chiral catalysts in green asymmetric synthesis especially in the efforts producing pharmaceutically important agents. The new family members with new reactivity and selectivity would be worthwhile studying in the future.

\section{References and Notes}

1. Lin, G.-Q., You, Q.-D \& Cheng, J.-F., Eds. Chiral Drugs (Wiley-VCH, Weinheim, 2011).

2. Jacobsen, E. N. \& Finney, N. S. Synthetic and biological catalysts in chemical synthesis: how to assess practical utility. Chem. Biol. 1, 85-90 (1994).

3. Yoon, T. P. \& Jacobsen, E. N. Privileged chiral catalysts. Science 199, 1691-1693 (2003).

4. Zhou Q.-L., Ed. Privileged Chiral Ligands and Catalysts (Wiley-VCH, Weinheim, 2011).

5. Li, C.-J. Exploration of new chemical reactivities for sustainable molecular transformations. Chem 1, 423-437 (2016).

6. Nozaki, H., Aratani, T., Toraya, T., \& Noyori, R. Asymmetric ring opening of gem-dibromocyclopropanes leading to allenic hydrocarbons. Tetrahedron Lett. 9 , 2087-2090 (1968).

7. Trost, B. \& Dietsche, T. New synthetic reactions. Allylic alkylations asymmetric induction in allylic alkylations. J. Am. Chem. Soc. 95, 8200-8201 (1973).

8. Hoppe, D., Hintze, F. \& Tebben, P. Chiral lithium-1-oxyalkanides by asymmetric deprotonation; enantioselective synthesis of 2-hydroxyalkanoic acids and secondary alkanols. Angew. Chem. Int. Ed. 29, 1422-1424 (1990).

9. Kerrick, S. T. \& Beak, P. Asymmetric deprotonations: Enantioselective syntheses of 2-substituted (tert-butoxycarbonyl)pyrrolidines. J. Am. Chem. Soc. 113, 97089710 (1991). 
10. Denmark, S. E., Nakajima, N. \& Nicaise, O. J.-C. Asymmetric addition of organolithium reagents to imines. J. Am. Chem. Soc. 116, 8797-8798 (1994).

11. Campos, K. R., Klapars, A., Waldman, J. H., Dormer, P. G. \& Chen, C.-y. Enantioselective, palladium-catalyzed $\alpha$-arylation of $N$-Boc-pyrrolidine $J$. Am. Chem. Soc. 128, 3538-3539 (2006).

12. Genet, C., Canipa, S. J., O’Brien, P. \& Taylor, S. Catalytic asymmetric synthesis of ferrocenes and P-stereogenic bisphosphines. J. Am. Chem. Soc. 128, 9336-9337 (2006).

13. Burns, M., et al. Assembly-line synthesis of organic molecules with tailored shapes. Nature 513, 183-188 (2014).

14. Lin, W., Zhang, K.-F. \& Baudoin, O. Regiodivergent enantioselective C-H functionalization of Boc-1,3-oxazinanes for the synthesis of $\beta 2$ - and $\beta 3$-amino acids. Nat. Catal. 2, 882-888 (2019).

15. Shintani, R. \& Fu, G. C. Highly enantioselective desymmetrization of anhydrides by carbon nucleophiles: reactions of Grignard reagents in the presence of (-)sparteine. Angew. Chem. Int. Ed. 41, 1057-1059 (2002).

16. Brunner, H., Kagan, H. B. \& Kreutzer, G. Asymmetric catalysis. Part 153: Metalcatalysed enantioselective $\alpha$-ketol rearrangement. Tetrahedron: Asymmetry 14, 2177-2187 (2003).

17. Smrčina, M., Poláková, J., Vyskočil, S. \& Kočovský, P. Synthesis of enantiomerically pure binaphthyl derivatives. Mechanism of the enantioselective, oxidative coupling of naphthols and designing a catalytic cycle. J. Org. Chem. 58, 4534-4538 (1993).

18. Hu, G., Holmes, D., Gendhar, B. F. \& Wulff, W. D. Optically active ( $a R)$ - and $(a S)$-linear and vaulted biaryl ligands: deracemization versus oxidative dimerization. J. Am. Chem. Soc. 131, 14355-14364 (2009).

19. Mimoun, H., de Saint Laumer, J. Y., Giannini, L., Scopelliti, R. \& Floriani, C. Enantioselective reduction of ketones by polymethylhydrosiloxane in the presence of chiral Zinc catalysts. J. Am. Chem. Soc. 121, 6158-6166 (1999).

20. Togni, A. Asymmetric allylic alkylation catalyzed by palladium-sparteine complexes. Tetrahedron: Asymmetry 2, 683-690 (1991).

21. Jensen, D. R., Pugsley, J. S. \& Sigman, M. S. Palladium-catalyzed enantioselective oxidations of alcohols using molecular oxygen. J. Am. Chem. Soc. 123, 7475-7476 (2001).

22. Ferreira, E. M. \& Stoltz, B. M. The Palladium-catalyzed oxidative kinetic resolution of secondary alcohols with molecular oxygen. J. Am. Chem. Soc. 123, 7725-7726 (2001).

23. Yip, K.-T., Yang, M., Law, K.-L., Zhu, N.-Y. \& Yang, D. Pd(II)-catalyzed enantioselective oxidative tandem cyclization reactions. synthesis of indolines through C-N and C-C bond formation. J. Am. Chem. Soc. 128, 3130-3131 (2006).

24. Dai, H. \& Lu, X. Palladium (II)/2,2'-bipyridine-catalysed addition of arylboronic acids to $N$-tosyl arylaldimines. Tetrahedron Lett. 50, 3478-3481 (2009). 
25. Holder, J. C., et al. Mechanism and enantioselectivity in Palladium-catalyzed conjugate addition of arylboronic acids to $\beta$-substituted cyclic enones insights from computation and experiment. J. Am. Chem. Soc. 135, 14996-15007 (2013).

26. Mase, N., Tanaka, F. \& Barbas III, C. F. Synthesis of b-hydroxyaldehydes with stereogenic quaternary carbon centers by direct organocatalytic asymmetric aldol reactions. Angew. Chem. Int. Ed. 43, 2420-2423 (2004).

27. Phuan, P.-W., Ianni, J. C. \& Kozlowski, M. C. Is the A-ring of sparteine essential for high enantioselectivity in the asymmetric lithiation-substitution of $N$-Bocpyrrolidine? J. Am. Chem. Soc. 126, 15473-15479 (2004).

28. McNally, A., Haffemayer, B., Collins, B. \& Gaunt, M. J. Palladium-catalysed C-H activation of aliphatic amines to give strained nitrogen heterocycles. Nature 510, 129-133 (2014).

29. Dearden, M. J., Firkin, C. R., Hermet, J.-P. R. \& O'Brien, P. A readily-accessible (+)-sparteine surrogate. J. Am. Chem. Soc. 124, 11870-11871 (2002).

30. Sheikh, N. S., et al. An experimental and in situ IR spectroscopic study of the lithiation-substitution of $N$-Boc-2-phenylpyrrolidine and -piperidine: controlling the formation of quaternary stereocenters. J. Am. Chem. Soc. 134, 5300-5308 (2012).

31. Freund, M. \& Horkheimer, P. Zur kenntniss des Cytisins. Ber. Dtsch. Chem. Ges. 39, 814-825 (1906).

32. Ebner, D. thesis, California Institute of Technology (2009).

33. Rouden, J., Lasne, M.-C., Blanchet, J. \& Baudoux., J. (-)-Cytisine and derivatives: synthesis, reactivity, and applications. Chem. Rev. 114, 712-778 (2014).

34. Grygorenko, O. O. Radchenko, D. S., Colochnyuk, D. M., Tolmachev, A. A. \& Komarov, I. V. Bicyclic conformationally restricted diamines. Chem. Rev. 111, 5506-5568 (2011).

35. Michael, J. P. Indolizidine and quinolizidine alkaloids. Nat. Prod. Rep. 24, 191$222(2007)$

36. Jiang, C., Lu, Y. \& Hayashi, T. High performance of a palladium phosphinooxazoline catalyst in the asymmetric arylation of cyclic $N$-sulfonyl ketimines. Angew. Chem. Int. Ed. 53, 9936-9939 (2014).

37. Dawson, G. J., Frost, C. G., Williams, J. M. J. \& Coote, S. J. Asymmetric palladium catalysed allylic substitution using phosphorus containing oxazoline ligands. Tetrahedron Lett. 34, 3149-3150 (1993).

38. von Matt, P. \& Pfaltz, A. Chiral phosphinoaryldihydrooxazolesas ligands in asymmetric catalysis: Pd-catalyzed allylic substitution. Angew. Chem. Int. Ed. 36, 566-568 (1993).

39. Sprinz, J. \& Helmchen, G. Phosphinoaryl- and phosphinoalkyloxazolines as new chiral ligands for enantioselective catalysis: very high enantioselectivity in palladium catalysed allylic substitutions. Tetrahedron Lett. 34, 1769-1772 (1993).

40. Brunner, H., Obermann, U. \& Wimmer, P. Asymmetrische katalysen: XXXII. Enantioselektive phenylierung von cis-cyclohexan-1,2-diol und meso-butan-2,3diol. J. Organomet. Chem. 316, C1-C3 (1986). 
41. Izumi, Y., Imaida, M., Fukawa, H. \& Akabori, S. Asymmetric hydrogenation with modified Raney nickel. II. Studies on modified hydrogenation catalyst. III. Bull. Chem. Soc. Jpn. 36, 155-160. (1963).

42. Mase, N., et al. Organocatalytic direct asymmetric aldol reactions in water. J. Am. Chem. Soc. 128, 734-735 (2006).

43. Maheswaran, H., et al. Enantioselective nitroaldol (Henry) reaction using copper(II) complexes of (-)-sparteine. Chem. Commun. 4066-4068 (2006).

44. Wu, X.-Y., Xu, H.-D., Zhou, Q.-L.\& Chan, A. S. C. Enantioselective Heck-type hydroarylation of norbornene with phenyl iodide catalyzed by palladium/quinolinyl oxazolines. Tetrahedron: Asymmetry 11, 1255-1257 (2000).

45. Campello, H. R., et al. Unlocking nicotinic selectivity via direct $\mathrm{C}-\mathrm{H}$ functionalization of (-)-cytisine. Chem 4, 1710-1715 (2018).

\section{Acknowledgments:}

We thank Dr. Xinghua Jin (Instrumental Analysis Centre-IAC, SPST, Tianjin University) for conducting NMR analysis, Dr. Jun Xu (IAC, SPST, Tianjin University) for the structure determination of the ligand L3, Ms. Yan Gao for HRMS for all novel compounds. We thank Prof. Jay Siegel (Tianjin University) for fruitful discussions.

\section{Funding:}

National Natural Science Foundation Grant No. 21871207, 22073067 and 21672159

J\&K Scientific Ltd.

\section{Author contributions:}

Conceptualization: JH, YD

Methodology: JH, FZ, LL, CC, YL, GL, SH, MZ, YY, YZ

Investigation: JH, FZ, LL, CC, YL, GL, SH, MZ, YY, YZ

Theoretical calculation: YD, BL

Funding acquisition: JH, YD

Project administration: JH, YD

Supervision: JH, YD

Writing - original draft: JH, YY

Writing - review \& editing: JH, YY, CC, MZ, YD

Competing interests: Authors declare that they have no competing interests.

Data and materials availability: All data are available in the main text or the supplementary materials. 